\title{
El uso del portafolio electrónico en evaluación educativa en Medicina.
}

\section{The use of the electronic portfolio training in education evaluation in Medicine.}

\author{
Gabriel Gutiérrez Morales $^{\mathbf{1}^{*}}$, Melchor Sánchez Mendiola ${ }^{2}$ \\ 1 Profesor del curso de Neumología Pediátrica en el Instituto Nacional de Pediatría. \\ Universidad Nacional Autónoma de México (UNAM). Email: gmgabo@hotmail.com \\ 2 Profesor de la Facultad de Medicina de la Universidad Nacional Autónoma de México \\ (UNAM), Ciudad de México. \\ * Correspondencia: gmgabo@hotmail.com
}

Recibido: 12/09/2020; Aceptado: 20/10/2020; Publicado: 5/11/2020

\begin{abstract}
Resumen: La educación médica se encuentra en una profunda transformación, que exigirá a los docentes reconsiderar las prácticas habituales de enseñanza y desarrollar estrategias innovadoras para optimizar el aprendizaje de los estudiantes y residentes. Se ha llevado a cabo una búsqueda bibliográfica en las bases de datos PubMed y ERIC, entre los años 2000-2019. Ningún instrumento individual de evaluación educativa provee todos los elementos que se requieren para evaluar a un individuo, necesitándose analizar suficientes tareas y que sean representativas de los diferentes aspectos que comprenden el aprendizaje. El uso del portafolio electrónico ayuda a recoger la evidencia del progreso del aprendizaje del alumno con un propósito determinado, brindando al profesor las herramientas necesarias para una evaluación más amplia y variada. Para su uso se requiere tener en cuenta aspectos inherentes al profesor, estudiante y a su entorno para lograr los objetivos de las intervenciones educativas.
\end{abstract}

Palabras clave: Educación Médica, Portafolios, Evaluación, Pedagogía, e-portfolio

\begin{abstract}
Medical education is undergoing a profound transformation, which will require teachers to reconsider current teaching practices and develop innovative strategies to optimize student and resident learning. No single educational assessment instrument provides all the elements required to evaluate an individual; sufficient tasks representative of the different aspects that comprise learning need to be analyzed. The use of the e-portfolio helps to collect evidence of student learning progress for a given purpose, providing the teacher with the necessary tools for a broader and more varied assessment. Its use requires taking into account aspects inherent to the teacher, student and their environment in order to achieve the objectives of educational interventions.
\end{abstract}

Keywords: Medical Education, e-portfolio, Evaluation, Asessment

\section{Introducción}

La educación médica se encuentra en medio de una gran transformación que exigirá a los docentes médicos reconsiderar las prácticas habituales de enseñanza, así como desarrollar estrategias innovadoras para optimizar el aprendizaje de los estudiantes y residentes. El conocimiento y la comprensión de las principales teorías de aprendizaje pueden facilitar la práctica del 
docente, ya que proporcionan una base racional para la selección de las estrategias de enseñanza específicas, construir los objetivos de aprendizaje y finalmente facilitar la aplicación de estrategias de evaluación que se cumplan con los objetivos propuestos (1).

En educación médica es importante retomar dos momentos importantes a lo largo de la historia: el primero, el denominado "Informe Flexner" de 1910, y el segundo, la II Declaración de Edimburgo de 1993. Estos dos documentos tuvieron un fin en común, generar las condiciones y directrices necesarias para educar a los estudiantes de medicina y futuros profesionales de la salud. La educación médica es referida como el proceso de formación de habilidades, destrezas, actitudes y responsabilidades de los médicos, mediante la integración progresiva de conocimientos y experiencias a lo largo de su formación (2).

La estructura del proceso educativo en la que se forman los médicos durante la enseñanza escolarizada de pre y posgrado está construida de hitos de evaluación, los cuales tienen que ser acreditados para poder ser promovidos a un grado mayor, lo que va formando el currículo del estudiante, percibido por éste como un pase o una sucesión de exámenes que parecen ser infinitos a lo largo del proceso (3). En el proceso educativo uno de los puntos más importantes es la evaluación del aprendizaje, definida por Miller en 2012 como un término genérico que incluye un rango de procedimientos para adquirir la información sobre el aprendizaje del estudiante y la formación de juicios por parte del profesor en cuanto a este proceso (4). Otras definiciones lo señalan como el concepto que abarca un rango de procedimientos que nos ayuda a obtener información del aprendizaje del estudiante en todos sus componentes (conocimientos, habilidades, valores, creencias y hábitos), así como la capacidad de razonamiento y resolución de problemas que correspondan con su nivel de desarrollo profesional.

\section{Metodología.}

Se realizó una revisión sistemática de la literatura sobre portafolios electrónico en educación, con énfasis en ciencias de la salud en las plataformas Medline (pubmed) y ERIC, del 2000 al 2019. Con Identificación de palabras clave y términos $\mathrm{MeSH}$ (Medical Subject Heading): portfolio electronic AND evaluate competence, encontrando 82 referencias, de las cuáles se tomaron las 25 orientadas a la evaluación en medicina.

\section{La evaluación del proceso educativo.}

Desde el punto de vista de su objetivo, la evaluación se puede clasificar en sumativa y formativa. La primera es aquella compuesta por la suma de valoraciones efectuadas durante un curso o unidad didáctica y nos permite determinar el grado con el que los objetivos de éste han sido logrados, permitiéndonos darle una calificación al estudiante o aprobar una competencia (5). Por otra parte, la evaluación formativa es la que se utiliza para monitorear el progreso del aprendizaje y proporcionar realimentación al estudiante sobre sus logros, deficiencias y oportunidades de mejora. Las recientes reformas en educación médica han traído nuevos sistemas sobre el proceso de enseñanza-aprendizaje-evaluación que fomentan el aprendizaje reflexivo y el crecimiento profesional, donde se involucran los participantes directos (profesores y estudiantes) con el fin de formar profesionales 
competentes que puedan atender y resolver los problemas de salud de manera efectiva, eficaz y con calidad (6).

Ningún instrumento individual de evaluación por sí solo provee todos los elementos que se requieren para evaluar integral y completamente a un individuo, por lo que debemos analizar muestras suficientes de tareas representativas de todos los aspectos que comprenden el aprendizaje, para no tener una visión limitada y sesgada al momento de evaluar el desempeño de los estudiantes. El reto de la evaluación en educación médica es de extrema complejidad y exige que se aborde con responsabilidad y profesionalismo, lo cual implica un proceso sistemático de acopio de información para lo que debemos utilizar instrumentos que cumplan con los requisitos mínimos de calidad y que cumplan con criterios cuantitativos y cualitativos (7).

Conforme surgen cambios en las perspectivas de la educación en medicina, se hace indispensable modificar las herramientas de evaluación, en congruencia con los nuevos paradigmas educacionales (p. ej. acceso a la información a través de las nuevas tecnologías, sesiones interactivas a distancia). La selección de instrumentos y estrategias depende del propósito y contenido del curso, de las estrategias de aprendizaje, la habilidad del docente y las características propias del grupo. Deberá ser congruente con el objetivo de la evaluación de acuerdo con los programas académicos normados por las instituciones educativas, con el fin de que, al término de éstos, los alumnos logren las competencias establecidas.

\section{El portafolio en la Educación Médica.}

Se describe desde 1990 por el Royal College of General Practitioners en el Reino Unido el uso del portafolio como instrumento de evaluación. Existen diversas definiciones de portafolio que varían dependiendo del objetivo que cada autor les otorga a los aspectos que lo constituyen, sin embargo, la palabra portafolio etimológicamente proviene del vocablo francés portefeuille, que refiere a la cartera de mano para llevar papeles o libros. Este concepto surgió inicialmente en otros ámbitos, como las artes, la fotografía y la publicidad, en los que se requiere de la presentación de evidencias para valorar los conocimientos, las habilidades básicas y las posibilidades de logros que se presentan a través de un conjunto de trabajos representativos (8).

Paulson y Meyer (1991) definen el portafolio como una colección intencional de los trabajos de un estudiante, la cual exhibe sus esfuerzos, sus progresos y sus logros en una o más áreas. La colección debe ser el resultado de la participación intencional de los estudiantes en la selección de los contenidos de su portafolio, debe explicar los criterios utilizados para realizar esta selección y debe justificar los procesos de reflexión del estudiante (9). En 1992, Arter y Spandel observaron que el término portafolios estaba de moda; sin embargo vieron que no quedaba claro lo que implicaba o significaba en realidad, particularmente cuando se usaba en el contexto de la evaluación. Estos autores ofrecieron la siguiente definición: “...una colección de trabajos del estudiante que nos cuenta la historia de sus esfuerzos, su progreso y logros en un área determinada. Esta colección debe incluir la participación del estudiante en la selección del contenido del portafolio, las guías para la selección, los criterios para juzgar méritos y la prueba de su autorreflexión". Esta definición enfatiza la naturaleza del desarrollo del proceso evaluativo y da importancia a la implicación activa que deben tener los estudiantes al 
reconocer lo que saben y lo que pueden hacer. Otra dimensión significativa de dicha definición es el reconocimiento de la reflexión del estudiante sobre los procesos de aprendizaje necesarios durante su trabajo, así como la integración de la evaluación en la enseñanza y en el aprendizaje (10). La AMEE (Association for Medical Education in Europe) describe al portafolio como una colección de trabajos de los estudiantes que exhibe esfuerzos, progresos y logros en una o más áreas del estudiante; la participación de los estudiantes debe ser de manera activa en conjunto con el profesor en la selección de los contenidos, los criterios de evaluación y de la reflexión sobre el material mostrado en éstos. Estas definiciones implican que el propósito de la evaluación del estudiante guiará el contenido del portafolio (11).

Al aplicarse al ámbito de la educación médica, podemos hacer referencia a lo descrito por Thistlethwaite: “Un portafolio en educación médica es una colección de documentos que proporcionan las pruebas del aprendizaje y un análisis reflexivo de los acontecimientos documentados. El portafolio debería demostrar que el alumno ha alcanzado el nivel requerido para su nivel de formación", poniendo énfasis en que el portafolio debe estar centrado en el estudiante (12). Con esta diversidad de definiciones que comparten ciertas características, podemos afirmar que el uso del portafolio debe representar las evidencias del progreso del aprendizaje del alumno con un propósito determinado, que brindan al profesor las herramientas necesarias para una evaluación amplia y profunda, basándose en logros o competencias desarrollados por los alumnos, con oportunidad de reflexionar sobre ellos. Esto lo convierte en una metodología de enseñanza y evaluación alternativa a aquellas de corte puramente cuantitativo.

\section{Bases pedagógicas del portafolio.}

El uso del portafolio se puede relacionar con varias filosofías, modelos o teorías de la educación. Su sustento psicopedagógico se encuentra principalmente en las teorías cognitivista, constructivista y sociocultural, las cuáles pueden estar entrelazadas (figura 1).

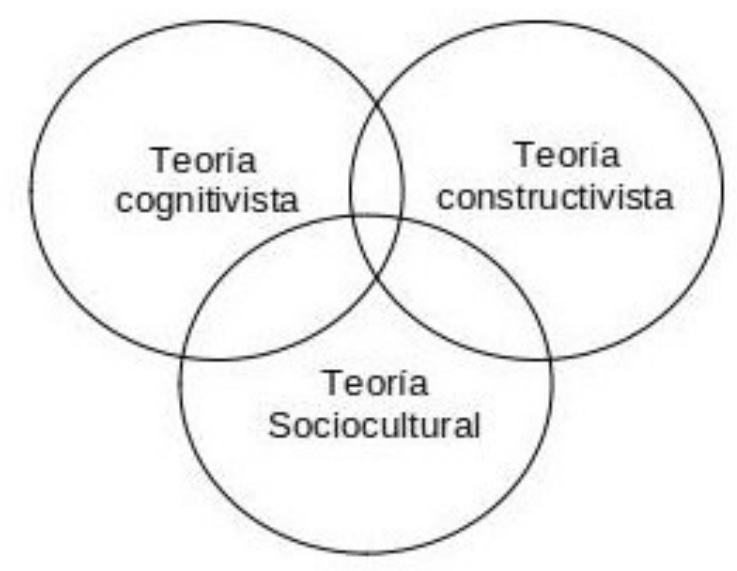

Figura 1. Sustento pedagógico del portafolio en evaluación educativa.

En el marco de la teoría cognitivista, el alumno utiliza herramientas cognitivas, tales como la comprensión, el procesamiento de la información, las percepciones y la memoria, para facilitar el aprendizaje asignado a los 
eventos. Se caracteriza por la creación de un aprendizaje significativo mediante el cual los estudiantes buscan comprender la estructura del conocimiento (1). En la visión de Ausubel, el aprendizaje significativo resulta de relacionar el nuevo conocimiento con lo que ya se conoce. Este enfoque aboga por la creación de estrategias de aprendizaje que vinculan conceptos de manera única para que el alumno entienda la estructura del conocimiento (13).

El constructivismo es un enfoque pedagógico que explica la forma en que los seres humanos se apropian del conocimiento. Esta teoría sostiene que el conocimiento no se descubre, se construye. Se fundamenta en las teorías de Piaget y Vigotsky, en las que cada persona debe edificar su propio conocimiento. Para esta teoría, el aprendizaje es un proceso activo, constructivo y orientado al logro de metas, cuyo cumplimiento depende del desarrollo de los procesos mentales superiores del alumno. Esta concepción implica que los sujetos del proceso modifiquen sus roles, modificando el paradigma tradicional (14).

El paradigma sociocultural fue una propuesta de reconocer los procesos psicológicos como resultado de la interacción del individuo con su medio social y cultural en un momento históricamente determinado, tal y como lo describe Vygotsky, afirmando que la interacción comunicativa es inherente a la condición humana lo cual se propone con el portafolio.

\section{Función del portafolio en la Educación Médica.}

La función principal de los portafolios es documentar lo que el alumno ha aprendido, de acuerdo con los objetivos curriculares específicos, para lo que es preciso que los contenidos que se incluyen en el instrumento reflejen el conocimiento y las habilidades definidas (tabla 1).

- Determinar los objetivos curriculares que deben alcanzarse mediante el portafolio.

- Establecer qué decisiones se tomarán sobre la base de las evaluaciones de los portafolios.

- Diseñar las tareas de evaluación de acuerdo a los objetivos curriculares.

- Definir los criterios para cada tarea de evaluación y establecer las pautas de rendimiento para cada uno de ellos.

- Determinar quién evaluará los ítems del portafolio.

- Capacitar a los docentes para calificar las evaluaciones.

- Enseñar de acuerdo al currículo, implementar las evaluaciones descritas y reunirlas en el portafolio.

- Evaluar el portafolio.

Tabla 1. Pasos básicos para el desarrollo de un sistema de evaluación. (Adaptado de Cataldi \& Lage (14).

Por su parte, la AMEE señala las características mínimas que constituyen un portafolio (11):

1. Contar con un carácter diagnóstico, de intervención, retroalimentación y aprobación (evaluación formativa y sumativa).

2. Contener evidencia descriptiva y logros de aprendizaje (información cuantitativa y cualitativa). 
3. Debe ser personal, ya que permite el rol activo del estudiante en los procesos de selección, análisis y reflexión de la evidencia.

4. Debe existir una adecuada y objetiva instrucción, definición de tareas y criterios de evaluación, por parte del facilitador, para asegurar su confiabilidad y validez.

5. Debe incorporar el conocimiento previo, para establecer las bases de la integración, favoreciendo el aprendizaje profundo y permitiendo la valoración de su utilidad más allá de la evaluación.

6. Permite una evaluación válida, confiable y practicable, siempre y cuando su aplicación esté bien planificada.

Driessen señala que el portafolios, para ser efectivo, debe contener una introducción y orientación adecuadas, integrar el contexto y los procedimientos, suministrar información a estudiantes y docentes, proveer pautas claras que no restrinjan la libertad de los estudiantes, al tiempo que sean de uso fácil y que no incluyan demandas que sobrepasen el tiempo limitado de estudiantes y mentores (16).

En las últimas décadas la incorporación de las tecnologías de la información y la comunicación (TIC) y la enseñanza mediante entornos virtuales, han favorecido la proliferación de experiencias y estudios centrados en el uso del e-portafolio, portafolio electrónico o digital, que facilitan su uso y aprovechamiento. Powers y cols. consideran que el surgimiento de los portafolios electrónicos tiene una serie de ventajas importantes, como que el material puede ser capturado, organizado, guardado y presentado en formato digital en una gran variedad de dispositivos y plataformas (17). Los elementos del portafolio electrónico pueden ser además enlazados a otros y presentar detalles adicionales. Actualmente un número cada vez mayor de escuelas de medicina utiliza portafolios electrónicos, ya que se pueden insertar hipervínculos para establecer conexiones entre la evidencia y las reflexiones. Los hipervínculos también pueden ser útiles para hacer una tabla de contenidos. Los profesores pueden navegar a través de esta lista de subtítulos, obtener una visión general rápida de todas las pruebas y hacer clic en la evidencia que es relevante para su propósito específico (18).

El reto de la educación médica es la formación integral de profesionales capaces de ejercer la práctica de la medicina aplicando conocimientos científicos actuales, realizar adecuadamente procedimientos técnicos y clínicos, demostrar facetas éticas y humanísticas, actualizarse permanentemente y participar en la investigación para la generación de conocimientos nuevos. Sin embargo, la evaluación de estos conocimientos, habilidades y destrezas en la práctica cotidiana requiere contar con herramientas que nos ayuden a evaluar de manera objetiva y sistematizada estas competencias, lo que ha dado paso al desarrollo de instrumentos como el portafolio.

A través de esta herramienta podemos hacer que el modelo educativo en la formación del médico se sustente en el desarrollo de competencias cognitivas (saber), metodológicas (saber cómo, demostrar cómo y hacer) y actitudinales (saber ser), que expresen los niveles de formación de la pirámide del conocimiento de Miller, para contribuir al desarrollo de la capacidad de análisis-síntesis, la creatividad y el pensamiento reflexivo y crítico, así como la implicación del propio autor en el proceso de aprender a aprender y de 
evaluación. En algunos países, el portafolio es utilizado de manera sistemática como parte de las evaluaciones de estudiantes de medicina y postgrado en la práctica, ya que logra identificar a médicos con bajo desempeño y garantiza un sistema en el que éstos participan activamente en la identificación y el cumplimiento de sus propias necesidades de aprendizaje. La evaluación del desempeño tiene dos propósitos: mejorar la calidad de la práctica de la medicina y asegurar que se haya cumplido un estándar mínimo de desempeño (19).

Swallow refiere que el portafolio electrónico estimula al alumno a que se responsabilice en el desarrollo de sus competencias y adopte una actitud más activa sobre su aprendizaje (20). De la misma manera, Mathers comprobó que con el uso del portafolio, los alumnos dedican más horas a la reflexión con lo que pueden mejorar la resolución de los problemas clínicos (21). Miranda, de la Pontificia Universidad Católica de Chile, señala que el portafolio electrónico permite un registro de actividades sistemático, así como un análisis del proceso de formación y la capacidad de enfocar el aprendizaje a las áreas en que las evaluaciones arrojan resultados insuficientes (22). Arnau y colaboradores refieren que, a través del uso del portafolio, se permite al profesor evidenciar algunos elementos clave en el proceso formativo y en el proceso profesional del residente, estimulando la utilización de estrategias de reflexión y autoaprendizaje con el fin de lograr las competencias evaluadas (23). En el Instituto Nacional de Pediatría de la Ciudad de México, recientemente publicamos el desarrollo y uso de un portafolio electrónico para la evaluación de competencias en residentes de neumología pediátrica, encontrando resultados positivos con su uso (24).

El portafolio electrónico también tiene desventajas ya que exige cierto nivel de conocimientos informáticos por parte del docente y de los alumnos que si no se poseen puede hacer que sea una herramienta poco práctica. Es necesario considerar que optar por el uso de este instrumento de evaluación, requiere de tiempo y dedicación del profesor para la definición y el diseño de las actividades y para la retroalimentación oportuna y constante, ya que sin ella puede disminuir el interés en la elaboración de estas (25).

\section{Conclusiones}

- El empleo del portafolio electrónico como herramienta en el proceso de enseñanza y evaluación permite dar seguimiento a los avances de los estudiantes, a la vez que éstos visualicen y reflexionen sobre su propio avance en el logro de sus competencias.

- Para el desarrollo del portafolio electrónico debemos tomar en cuenta las diversas plataformas en el mercado, las habilidades de los alumnos y del profesor para el manejo de la plataforma elegida, las características técnicas de la tecnología a utilizar, acceso de los estudiantes al medio, costos, control del estudiante sobre dicho medio y la facilidad del manejo para el profesor, con el fin de que el producto cuente con las características indispensables para lograr los objetivos planteados.

\section{Referencias}

1. Torre D. Overview of Current Learning Theories for Medical Educators. Am J Med. 2006;119(10):903-7. http://doi.org/10.1016/j.amjmed.2006.06.037. 
2. Sanchez, M. Lifshitz A. (2015). Educación Médica. Teoria y practica. Primera edición. México, D.F. Elsevier. https://www.elsevier.com/books/educacion-medicateoria-y-practica/sanchez-mendiola/978-84-9022-778-7

3. Shumway JM, Harden RM. AMEE guide no. 25: The assessment of learning outcomes for the competent and reflective physician. Med Teach. 2003;25(6):56984. http://doi.org/10.1080/0142159032000151907.

4. Miller MD, Linn RL, Gronlund NE. (2013). Measurement and Assessment in Teaching (11으. ed.). Londres: Pearson. https://www.pearson.com/us/highereducation/program/Miller-Measurement-and-Assessment-in-Teaching-11thEdition/PGM30380.html

5. Flores-Hernández F, Contreras-Michel N, Martínez-González A. Evaluación del aprendizaje en la educación médica. Rev. Fac. Med. 2012; 55(3):42-48. http://www.scielo.org.mx/scielo.php?script=sci arttext\&pid=S0026$17422012000300008 \& \operatorname{lng}=$ es.

6. Miller A., ArcherJ. Impact of workplace based assessment on doctors education and performance: a systematic review. BMJ 2010;341: c5064. https://doi.org/10.1136/bmj.c5064

7. Holmboe ES, Sherbino J, Long DM et al. The role of assessment in competencybased medical education. Med Teach 2010;32(8):676-82. http://doi.org/10.3109/0142159X.2010.500704.

8. Sánchez RB, Barragán Sánchez R. El Portafolio, metodología de evaluación y aprendizaje de cara al nuevo Espacio Europeo de Educación Superior. Una experiencia práctica en la Universidad de Sevilla. Rev Latinoam Tecnol Educ. 2005;4(1):121-40.

https://mascvuex.unex.es/revistas/index.php/relatec/article/view/189

9. Paulson FL, Paulson PR, Meyer CA. What Makes a Portfolio a Portfolio? $\begin{array}{lllll}\text { Educational } & \text { Leadership. } & \text { 1991; } & 48 & \text { (5): }\end{array}$ https://web.stanford.edu/dept/SUSE/projects/ireport/articles/e-portfolio/what \%20makes\%20a\%20portfolio\%20a\%20portfolio.pdf

10. Klenowski V. 2014. Desarrollo de portafolios. Primera edición. Editorial Narcea, España. https://narceaediciones.es/es/educacion-hoy-estudios/187-desarrollo-deportafolios-para-el-aprendizaje-y-la-evaluacion-9788427714489.html

11. David MFB, Davis MH, Harden RM et al. AMEE Medical Education Guide No. 24: Portfolios as a method of student assessment. Med Teach. 2001;23(6):535-51. http://doi.org/10.1080/01421590120090952.

12. Thistlethwaite JE. How to keep a portfolio. Clin Teach. 2006;3(2):118-23. https://doi.org/10.1111/j.1743-498X.2006.00078.x

13. Ausubel DP, Novak JD, Hanesian H. 1983. Psicologia educativa: Un punto de vista cognitivo. Editorial Trillas. https://www.worldcat.org/title/psicologia-educativa-unpunto-de-vista-cognoscitivo/oclc/970597155

14. Ortiz D. Constructivism as theory and teaching method. Sophia. 2015;19(2):93110. https://dialnet.unirioja.es/descarga/articulo/5973095.pdf

15. Cataldi Z, Lage FJ. E-Portafolio. Una Opción Metodológica más auténtica para la Evaluación de aprendizajes autónomos en educación superior. 2010;10.https://core.ac.uk/download/pdf/296346444.pdf

16. Driessen E, Van Tartwijk J, Van Der Vleuten C, Wass V. Portfolios in medical education: Why do they meet with mixed success? A systematic review. Med Educ. 2007;41(12):1224-33. http://doi.org/10.1111/j.1365-2923.2007.02944.x.

17. Powers, D., Thomson, S. y Buckner, k. (2000). Electronic Portfolios. En BULLOCK, A.A. y HAWK, P.P. (2000). Developing a teaching portfolio. A guide for preservice and practicing teacher. Ohio. Merrill-Prentice-Hall. https://www.semanticscholar.org/paper/Developing-a-Teaching-Portfolio\%3A-A- 
Guide-for-and-Adams-Bullock-Hawk/ c33bbea094c5b0a3bb9cf0f8fa67834442cead67

18. Van Tartwijk J, Driessen EW. Portfolios for assessment and learning: AMEE Guide no. 45. Med Teach. 2009; 31 (9): 790-801. http://doi.org/10.1080/01421590903139201.

19. Wilkinson TJ, Challis M, Hobma SO, et al. The use of portfolios for assessment of the competence and performance of doctors in practice. Med Educ. 2002;36(10):918-24. http://doi.org/10.1046/j.1365-2923.2002.01312.x.

20. Swallow V. Learning in practice: But who learns from who? Nurse Educ Pract. 2006;6(1):1-2. http://doi.org/10.1016/j.nepr.2005.11.001.

21. Mathers NJ, Challis MC, Howe AC, Field NJ. Portfolios in continuing medical education- Effective and efficient? Med Educ. 1999;33(7):521-30. http://doi.org/10.1046/j.1365-2923.1999.00407.x.

22. Miranda P, de la Fuente R, de la Cuadra JC, Corvetto M, Altermatt F. Portafolio electrónico en residencia de subespecialidad en anestesia regional y analgesia perioperatoria. Investig en Educ Médica 2015;4(14):e7. Available from: http://dx.doi.org/10.1016/S2007-5057(15)30037-5

23. Arnau I Figueras J, Torán MP et al. Introducción del portafolio formativo como instrumento de tutorización de residentes de Medicina Interna: Revisión de una experiencia piloto, 2005-2006. Rev Clin Esp 2008;208(9):447-54. Available from: http://dx.doi.org/10.1157/13127606

24. Gutiérrez G. Evaluación de competencias en residentes de neumología pediátrica: uso del portafolio electrónico. Neumol y Cir Torax Vol 78 - Núm 14-9 Enero-marzo 2019. https://dx.doi.org/10.35366/NT191A

25. Ramírez Lopez NL. Portafolio electrónico en educación médica y las herramientas de la web para su elaboración. Inv Ed Med 2013;2(8):225-228. http://doi.org/10.1016/S2007-5057(13)72716-9.

(C) 2020 por los autores. Enviado para su publicación en acceso abierto bajo los términos y condiciones de la licencia Creative Commons Attribution (CC BY) (http://creativecommons.org/licenses/by/4.0/). 